\title{
DIE ANALYSE DER AUFFÜHRUNG DES THEATERSTÜCKES "DIE MUTTER COURAGE UND IHRE KINDER" VON BERTHOLT BRECHT VON DEUTSCHSTUIERENDEN AN DER UNIMED IM JAHRGANG 2017-2018
}

\author{
Hartoni \\ Ahmad Bengar Harahap \\ Tanti Kurnia Sari
}

\begin{abstract}
AUSZUG
Das Ziel dieser Untersuchung ist es, die intrinsische Aspekte des Theaters in der Aufführung "Die Mutter Courage und ihre Kinder" von Bertholt Brecht von Deutschstudierenden an der Unimed im Jahrgang 2017-2018 zu analysieren. Die Datenquelle in dieser Untersuchung sind die Aufführung des Theaters des LKKTheaters. Die Daten der Untersuchung ist Dialogen und Szene die intrinsische Aspekte des Theaters sind. (1) die Charakaterisierung sind Protagonisten (Die Mutter, Katrin, Fejos) Antagonisten sind (Eilip, der schwarze Soldat, der weisse Soldat), (2) Einstellung des Theaters istim Oktoberfest an der Unimed gemacht. Die Theateraufführung findet vor der Bühne statt. Die Bühne ist im Hofvon FBS Unimed, (3) Perspektive des Theaters ist Die Perspektive des Theater ist dritte Person. Die dritte Person ist die Person zwischen zwei Sprecher bei dem Komunikation gesprochen wird, (4) Sprachstil ist Pleonasme,Sinekdok, und die Schritte, (5) das Erzählungsverlauf ist Erzählvorstellung, Konflikt, Konflikte mit den viele Proben, und Klimaks. (6) Thema ist die Mutter, eine starke und standhaffe, die so viele Probeleme in ihrem Leben hat. Lehre ist starr und geduldig erhalt die Prüfung, obwohl die grosse Probleme sind.

Dieser Tätigkeit erscheint manchmal in der Szenen und Dialogen von dem Theater "Die Mutter Courage und ihre Kind " von Bertholt Brecht. Die Ergebnisse der Untersuchung können die Erkenntnisse die instrisische Aspekte des Theatersdarstellen
\end{abstract}

Schlüsselwörter : Aufführung des Theaters.

\section{EINLEITUNG}

Das Theater ist eine Lebensimitation auf der Bühne. Durch das Theater können die den Mandie tatsächlichen Ereignisse in der Gesellschaft sehen. Manchmal ist der Konflikt in dem Theater gleich mit dem Konflikt der Gesellschaft. Das Drama ist ein Bild der Weltanschaung, Freude und Leid,(Waluyo, 2002:1)

Das Theater kann die Auffuhrüng realisieren, es gibt auch Theater, die als Lernmedium benutzt werden. Die Aufführung kann für den Literaturunterricht 
dienen. Der Literaturunterricht lehrt Kunst, Poesie, und Drama. Normalerweise lernen aber die Studenten nur Theorien, deshalb werden durch die Untersuchung die neuen Lernmaterialien vorgestellt. Durch eine Aufführung des Theaters lernen die Studenten viel über Literatur. In Deutschland wird die Aufführung darstellende Kunst genannt. Für die darstellende Kunst müssen viele Vorbreitungen getroffen werden, wie Musik, Schmuck, Costüm, usw. Sie helfen den Zuschauern des Theaters die Aufführung verstehen.

Das Theater hat auch einige wichtige inhaltliche Teile, wie die Struktur oderintrinsischeAspekte. In der Struktur gibt es die Charakterisierung, Einstellung des Theaters, die Perspektive des Theaters, Sprachstil, das Erzählungsverlauf, Thema und Lehre. Alle Teile sind wichtige Teile im Theater. Durch die Struktur können die Zuschauer die Erzählung verstehen. Diese Teile werden in dieser Untersuchung untersucht.Das Theater, das in dieser Untersuchung analysiert wird, ist “ Die Mutter Courage und ihre Kinder”, das von dem LKK Theater aufgeführt wird. Deshalb ist diese Novelle ein guter Lernstoff und sie kann interessanter werden, wenn sie aufgeführt wird.

In dieser Untersuchung wählt der Verfasser die intrinsische Aspekte der Aufführung des Theaterstückes "Die Mutter Courage und ihre Kinder" zu analysieren. Diese Untersuchung benutzt die Theorie von Aminuddin (2008:142).

\section{THEORETISCHE GRUNDLAGE \\ Der Begriffder Aufführung}

Sedyawati (2006 :59) stellt fest, dass die Spuren der indonesischen Aufführung in prähistorischen Zeiten vor allem in der Bronzezeit begannen wurde. Es wurde mit der Entdeckung einiger zeitgenössischer Metalle bewiesen, die Aufführungen von Menschen enthalten, die Kopfschmuck mit langen Federn und Masken tragen. Schriftliche Quellen zeigen, dass die Reliefs am Tempel die Tanz-Szene deutlich zeigen. Verschiedene literarische Werke zu dieser Zeit verstärken auch die Entwicklung der Performancekunst.

\section{Der Begriffder Aufführung}


Sedyawati (2006 :59) stellt fest, dass die Spuren der indonesischen Aufführung in prähistorischen Zeiten vor allem in der Bronzezeit begannen wurde. Es wurde mit der Entdeckung einiger zeitgenössischer Metalle bewiesen, die Aufführungen von Menschen enthalten, die Kopfschmuck mit langen Federn und Masken tragen.

Nach Dawn (2009 : 15) ist eine Aufführun eine sichtbare (physikalische) Form, Form ist etwas, das vor uns ist, damit es gesehen und berührt werden kann. In Zusammenhang mit Kunst.

Nach Dawn (2009 : 15) ist eine Aufführun eine sichtbare (physikalische) Form, Form ist etwas, das vor uns ist, damit es gesehen und berührt werden kann. In Zusammenhang mit Kunst.

Seodarsono (2003: 1) behauptet, dass die Aufführung ein Zweig der Kunst ist, der immer im Leben der Gesellschaft erscheint. Die Aufführung trägt einige Teile der traditionellen Kunstform, und hat die Mission, die Publikum vermittelt werden möchte.

\section{Der Begriff des Theaters}

Budianta und andere (2002:95) stellen fest, dass das Theater einliterarisches Genre ist, dessen physisches Aussehen mit Dialogenund Figuren in der Szene gezeigt wird. In derTheater-Aufführung treten der Dialog oder die Gesprächeauf der Bühne auf, denn der Dialog bestimmt den Inhalt der darstellenden Theater-Geschichte.

Waluyo (2002:1) meint, das Theater ist eine Lebensimitation auf der Bühne sein. Durch das Theater können die Zuschauer die wircklichen Ereignisse in der Gesellschaft sehen. Manchmal ist der Konflikt im Theater gleich mit dem Konflikt in der Gesellschaft. Astone und Savona (in Dewojati, 2010: 22) behaupten, dass das Theater eine Dialogeinstellung von einem Hauptext (der Dialogtexte der Figuren) und Nebentexten (Anweisung der Aufführung) ist.

\section{Die Theorien intrinsische Aspekte des Theaters}

Aminuddin (in Wahyudi 2008: 142) stellt fest, das Theater eine Erzählung ist, die von Schauspielern gespielt wird, und in dem die neue Phantasie in einer Erzählung gemacht wird. Die Struktur des Theaters nach Aminuddin ist : 


\section{1. die Charakterisierung}

Der Figur, von der das Ereignis in der fiktiven Geschichte getragen wird, wird Charakterisierung genannt.

A. Der Protagonist ist der Figur, der die Geschichte unterstützt, der Figur wird meistens als gute Person dargestellt. Normalerweise ist diese Charakter gut und positve Gedanken haben, wie zum Beispiel großzügige, ehrliche, demütige, Verteidiger,clevere, unabhängige und loyale Freunde.Diese Anzahl ist in der Regel ein gutes Zeichen. Der Protagonist wird sehr Zuschauer gelieben.

B. Der Antagonist ist der Figur, der meistens als unfreundlich oder bösere Person dargestellt wird. Normalerweise ist dieser CharakterGewöhnlich wird diese Figur als ein Charakter von schlechtem Charakter und negativ, wie arrogant, eifersüchtig und ehrgeizig dargestellt.

2. Einstellung des Theaters

Einstellung ist Platzt, Zeit oder Ereignis, und hat psyikalische und pshychologische Funktion. Aber Leo (in Aminuddin 1998:64) behauptet, dass der Hintergrund nicht nur den Platzt, die Zeit, die Ereignisse, die Atmosphäre oder die Dinge in der Aufführung sondern auch die Atmosphäre der Einstellung des Gedankens, und des Lebenstils.

\section{Die Perspektive des Theaters}

Die Darstellungsart zeigt der Schauspieler in der Geschichte, die sie beschreiben Bei der Bestimmung der Sichtweise kann man sehen, wie der Autor oder Erzähler die Geschichte beschreibt, die stattgefunden hat. In diesem Fall wird das Theater direkt von einem Schauspieler oder Spieler gespielt, der die Geschichte erzählt.

\section{Sprachstil}

Die Darstellungart bringt die Idee mit dem Pleonasme, Sinekdok, und die Schritte. Sprachstil in der Geschichte kann aus der Art und Weise gesehen werden, wie Spieler in jeder Konversation oder Geste Wortwahl verwenden, die fest, konnotativ oder schön ist.

a. Pleonasme des Sprachstils ist Sprachstil in einem klaren Dialog, aber gibt es Sätze oder Schrite der Steigerung für Dialog erklären. 
b. Sinekdok des Sprachstils ist wenige Teile in dem Dialog mit der allen Steigerung erklären

c. Die Streit des Sprachstils ist normalerweise Sprachstil anders zwischen Dialog und das Faktum benutz wird

5. Das Erzählungsverlauf

Der Erzählungsverlauf funktioniert als das Erzählungsskellet. Für Zuschauer, das Erzählungsskellet ist ein Verständnis bei der alle Erzählung klar und reihe sind.

6. Thema und Lehre

Die Idee, die eine Geschichte begründet, ist ein Thema, und das Thema dient als Grundlage des Autors im Werk

\section{Semantische Theorie von Pateda}

1. Verständnis

Die Bedeutung ist gleich mit dem Thema. Die Bedeutung kann erreicht, wenn zwischen Sprecher mit andere Sprecher kommunizieren .

2. Das Gefühl

Das Gefühl ist einige gemeinsam mit der Bedeutung. Wenn Sprecher etwas mit den anderen Sprecher sagt, hat die Wörter oder Satz die Bedeutung oder das Gefühl. Sodass jeder Wort hat die gleiche Bedeutung mit dem Gefühl.

3. Der Ton

Der Ton ist Verbindung mit der Bedeutung und dem Gefühl. Wenn Sprecher mit dem höhen Ton spricht, zeigt es Unfreude..

4. Das Ziel

Wenn Sprecher mit dem anderen Sprecher spricht, hat er das Ziel von der Wörter ihn sprechen. zB, "es ist regnet" es zeigt das Ziel, dass wir nicht gehen. Das Wetter ist nicht gut, es ist regnet.

Die Synopsise des Dramas "Die Mutter Courage und ihre Kinder"

In der Novelle Günter Busch, 2016. Bertholt Brecht Mutter Courage und ihre Kinder. Frankfurt am Main erzählt, Diese Novelle ist gute Novelle in Deutschland am 1939. Mit der Mut und Geduld der Mutter macht den Mensch berührt in diser Erzählung fühlen. Es kann Beispiel für den Mensch werden. dass 
der Man wird, muss geduldig sein. Die Handlung im Dreißigjährigen Krieg über viele Jahre hinweg spielt. Die Marketenderin, Anna Fierling, auch Mutter Courage genannt, zieht mit ihrem Planwagen, ihren beiden Söhnen, dem mutigen Eilif, dem ehrlichen, aber dummen Fejos und ihrer stummen Tochter Kattrin durch die Länder. In Südschweden kommt der Mutter Courage ein Kind abhanden. Eilif wird nämlich von einem Feldwebel für den Krieg angeworben. Zwei Jahre später trifft sie ihren Sohn Eilif als Helden in Polen wieder. Nach weiteren Jahren wird ihr jüngster Sohn Fejos Zahlmeister und verwaltet die Regimentskasse.

\section{Die Organisation von dem LKK-Theater}

Das LKK-Theater der UNIMED wurde am 1988 gegrundet, und es existiert noch bis jetzt. Das LKK-Theater ist das Medium für die Aktivitäten der Stundenten in der darstellenden Kunst an der UNIMED. Das LKK-Theater liegt in der Olahraga Strasse Nummer 10 an der UNIMED. Das LKK-Theater hat auch ein Motto, das heißt von Uns durch Uns für Uns, LKK-Theater huuu'.

\section{Die konzeptuellen Grundlagen}

Eine Novelle kann auch auffgeführt werden. Eine Aufführung der Novelle kann interessanter sein, und den Lesern leichten machen, der Inhalt und die Lehre $\mathrm{zu}$ verstehen. Eine Aufführung kann als ein Lernmedium besonders im Literaturfach benutzt werden. Es gibt viele Dinge, die mit einer Aufführung gelernt werden wie die strukturische Elemente. Die Theater Theorien von Aminuddin erklärt die instrinsische Aspekte des Theaters. Die instrinsische Aspekte des Theaters enthält die Charakterisierung, Einstellung des Theaters, die Perspektive des Theaters, Sprachstill, Erzählungsverlauf, Thema und Lehre.

\section{UNTERSUCHUNGSMETHODE}

In dieser Untersuchung wird die deskriptive Methode verwendet, um die intrinsischeAspekte des Theaters "Die Mutter Courage und ihre Kinder" des LKK-Theaters $\mathrm{zu}$ analysieren.Die Daten in dieser Untersuchung sind die Aufführung des Theaters "Die Mutter Courage und ihre Kinder" und an der Bibliothek UNIMED durch geführt. Die Datenquelle der Untersuchung ist die Dialogen und Szene on der intrinsische Aspekte des Theaters 
In dieser Untersuchung werden die Daten aus verschiedenen Dokumentationen und wissenschaflicher Literatur gesammelt, wie ;

Um die Daten zu bekommen, werden folgenden Schritte unternommen:

1. Die Aufführung des Theaters "Die Mutter Courage und ihre Kinder" anschauen

2. Die insrinsische Aspekte der Aufführung des Theaters "Die Mutter Courage und ihre Kinder" markieren

3. Die Datentriangulation

4. Die intrinsische Aspekte der Aufführung des Theaters "Die Mutter Courage und ihre Kinder" von Aminuddin Theorien analysieren

5. Die Analyse beschreiben

6. Durch Experten prüfen lassen

7. Eine Zusammenfassung schreiben

\section{Datenbeschreibung}

Die Daten dieser Untersuchung werden der Aufführung das Theaters UNIMED "Die Mutter Courage und ihre Kinder" aus dem Jahr 2017 von den Deustchstudierenden entnommen. Die Aufführungvergangenheit 12 Minuten lang.Im Theater "Die Mutter Courage und ihre Kinder"lassen sicheinige intrinsische Aspekte des Theatersfinden. Danach werden diese Daten rausgesucht und analysiert. Nach der Analyse wird es gefunden, dass es in dem Theater "Die Mutter Courage und ihre Kinder" von Bertholt Brecht von LKK-Theater sechs intrinsische Aspekte des Theaters gibt.

Die intrinsischen Aspekte des Theaters sind nach Aminudiin (in Wahyudi 2008:142) folgende.

1. Die Charakterisierung

2. Einstellung des Theaters

3. Die Perspektiven des Theaters

4. Sprachstil

5. Der Erzählungsverlauf 


\section{Thema und Lehre}

Die Analyse der sechs intrinsischen Aspekte im Theater "Die Mutter Courage und ihre Kinder" von Bertholt Brecht werden folgendermassen beschrieben.

\section{Die Charakterisierung}

a) Der Protagonist

(Dialog, 03:05)

Der schwarze Soldat :Oke. Ich bezahle das Essen, aber du müsst mit mir mitkommen

Die Mutter :Nein, läd nicht mein Kind ein (böse)!

Der schwarze Soldat:Dein Kind istfüht sich bei mir

Die Mutter :Nein, für immer nicht. Verstehst du ?(böse)

Eilip

:Es ist Okay Mutti, ich bin mir sicher, dass es eine nette Person ist. Du mus

Die Mutter nicht wütend sein

(Die Mutter)

:Aber Eilip (weinte) Eilip

Erklärung :Dieser Dialog des dicken Satzs zeigt die wütende Mutter, aber trotz ihrer Wut weint sie immer noch. Durch ihren Gesichtsausdruck und ihre Stimme,

b) Der Antagonist

(Dialog , 07:29)

Katrin

: Mutti, darf ich das sehen ? (bittet die Mutter um es zu sehen)

Die Mutter : Fejos (erschrak)

Der weiße Soldat :(schiesst Fejos plötzlich sofort vor den Augen der Mutter)

Fejos :Meine Mutti(sterben)

Der weiße Soldat :ist das dein Sohn ? bringt der Körper züruck

( Der weisse Soldat)

Erklärung: Dieser Dialog des dicken Satzsin der Szene kann man sehen dass der weiße brutale Soldat Fejos sofort schiesst, wenn 
die Mutter und Katrin von der Post der weißen Soldat vorbei kommen. In diesem Fall kann Man sagen, dass der weiße Soldat sehr sadistisch ist.

\section{Einstellung des Theaters}

Die Einstellung des Theaters befindet sich im Hof von FBS Unimed, in diesem Theater ist der Theaterplatz deutlich zu sehen.

\section{Die Perspektive des Theaters}

a. Die Perspektive des Theater ist dritte Person. Die dritte Person ist die Person zwischen zwei Sprechen bei dem Komunikation gesprochen wird.

(Dialog, 11:20)

\section{Katrin \\ Die Mutter}

\author{
:Mutti(schlafend) \\ :Katrin , Katrin, steh auf \\ Katrin steh auf (weinte) Ich \\ weiss Katrin, du schläfst, ja \\ du schläfst Katrin. ja, du \\ schlafst.( lachelnd und hört \\ traurig auf)ruhigbitte. Sie \\ schläft. \\ : (velassen die Mutter)
}

Erklärung : Dieser Dialog des dicken Satzs der Aufführung erzählte über die dritte Person, obwohl die dritte Person in dem gleichen Platzt ist.

\section{Sprachstil}

In der Aufführung gibt es drei Teile von dem Sprachstil

a. Pleonasme des Sprachstil ist Sprachstil in einem klaren Dialog, aber gibt es die Sätze oder Schritte der Steigerung für Dialog erklären.

b. Sinekdok des Sprachstil ist Sprachstil wenige Teile in dem Dialog mit der allen Steigerung erklären 
c. Die Streit des Sprachstil ist normalerweise Sprahstil anders zwischen Dialog und das Faktum benutzt wird.

\section{Der Erzählungsverlauf}

Der Erzählungsverlauf sind Erzählvorstellung, Konflikt, Konflikte mit den viele Proben, und Klimaks. Das kann Man auch dieses Erzählungsverlauf als Vorwärtshandlung (Plot) der Aufführung

a. Erzählvorstellung ist die Ereignis in der Erzählung mit der Charakterisierung, dem Ort, und der Arbeit vorstellen. Das kann man Erzählvorstellung bei dem Dialog sehen

b. Konflikt ist ein Problem bei Theater sein. Durch Konflikt macht Theater schön. Damit bei dem Theater nicht flach ist.

c. Konflikt mit der vielen Probe ist die Erzählung mit der viele Konflikt inhalt. Die Konflikt gibt es mit der einige Teile in dem Szene.

\section{Thema und Lehre}

Das Thema in dem Theater "Die Mutter Courage und ihre Kinder" ist dieMutter, eine starke und standhaffe, die so viele Probeleme in ihrem Leben hat.

Die Lehre in dieser Erzählung sind:
a. Bei den Familien arbeiten zussamen
b. Man muss fleissige Arbeit und keine Zurück in dem Leben
c. Starr und geduldig erhalt die Prüfung, obwohl die grosse Probleme sind.

\section{Diskussion}

In der Aufführung des Theaters "Die Mutter Courage und ihre Kinder" von Bertholt Brecht gibt intrinsische Aspekte. Die intrinsische Aspekte ist von Aminuddins Theorie, das sind die Charakterisierung, Einstellung des Theaters, Perspektive des Theaters, Sprachstil, Erzählungsverlauf, Thema und Lehre. Fur die Aufführung analysiert, benutzt Stützung der Theorie von Pateda. Mit der Theorie von Pateda kann Man gute Zusammenfassung machen. Die Theorie von Pateda sind Verständnisse, das Gefühl, die Bedeutung, der Ton, und das Ziel. Mit der 
Theorie von Pateda zusammenbinden mit Aminuddin's Theorizusammen, kann Man die Charakterisierung (den Protagonist und Antagonist), Sprachstil, Erzählungsverlauf wissen. Man kann das Gefühl von dem Szene oder Dialog einfügen.

\section{Die Schlussfolgerung}

1. Aufgrund der gemanten Untersuchungsergebnisse fallen die Schlussfolgerungen folgendermaßen aus: die Aufführung des Theaterstückes. Dieser Aufführung analysiert intrinsische Aspekte des Theaters. Es gibt 6 intrinsische Aspekte von Aminuddin, nämlich dieCharakterisierung, Einstellung des Theaters, die Perspektive des Theaters, Sprachstil, das Erzählungsverlauf, Thema und Lehre.

2. Nach der Untersuchung des Theaters "Die Mutter Courage und ihre Kinder"wird dieser Aufführung mit Aminuddin's Theorie analysiert. Diese Theorie wird von Pateda's Theorie stützt. Der Theorie von Aminuddin ist Charakterisierung, Einstellung des Theaters, die Perspektive es Theaters, Sprachstil, das Erzählungsverlauf, Thema und Lehre, und von Pateda's Theorie ist Verständnis, das Gefühl, der Ton, und das Ziel. Sodass Zusammenfassung sind: die Charakaterisierung sind Protagonisten (Die Mutter, Katrin, Fejos) Antagonisten sind (Eilip, der schwarze Soldat, der weisse Soldat), Einstellung des Theaters istDer Ort der Einstellung wurde im Oktoberfest an der Unimed gemacht. Die Theateraufführung findet vor der Bühne statt. Die Bühne ist im Hof von FBS Unimed, Perspektive des Theaters ist Die Perspektive des Theater sind dritte Person. Die dritte Person ist die Person zwischen zwei Sprecherbei dem Komunikation gesprochen wird, Spracstil sind Pleonasme des Spracstil, Sinekdok des Sprachstil, und die Schritte des Sprachstil, das Erzählungsverlauf sind der Erzählungsverlauf sind Erzählvorstellung, Konflikt, Konflikte mit den viele Proben, und Klimaks. Das kann Man auch dieses Erzählungsverlauf als Vorwärtshandlung (Plot) der Aufführung, Thema ist die Mutter, eine 
starke und standhaffe, die so viele Probeleme in ihrem Leben hat. Lehre sind :

a. Bei den Familien arbeiten zussamen

b. Man muss fleissige Arbeit und keine Zurück in dem Leben

c. Starr und geduldig erhalt die Prüfung, obwohl die grosse Probleme sind

\section{LITERATUR VERZEICHNIS}

Atmazaki, 1990. Pembacaan Karya Susastra Sebagai Suatu Seni Pertunjukan. Padang: Angkasa Raya Padang

Aminuddin (in Wahyudi 2008). Pengantar Teori Sastra. Jakarta: PT. Grasindo

Astone und Savana (in Dewojati, Cahyaningrum. 2010). Drama, Sejarah, Teori, Dan Penerapannya. Yogyakarta.GadjahMada University Press

Budianta (in Waluyo2002). StudiKasuspadaTeaterWadasFakultasDakwah IAIN WalisongoSemarang. Semarang: Institut Agama Islam Negeri Walisongo Semarang

Busch, Günter, 2016. Bertholt Brecht Mutter Courage und ihre Kinder. Frankfurt am Main: SV(Shurkamp Verlag)

Brüder, Grimm (in Janati 2014) Citra Tokoh Utama Perempuan dan Nilai Moral dalam Dongen "Schneewitchen und Rosenrot" dari Kumpulan Dongen und Kinder Und Hausmärchen Brüder Grimm, Yogyakarta : Universitas Negeri Yogyakarta

Kosasih, 2003. Ketatabahasaan Dan Kesusastraan. Bandung: PT. Yrama Widya

Pratiwi, Yuni. Siswiyanti, Frida. 2016. Teori Drama dan Pembelajaran, Yogyakarta: Penerbit Ombak

Waluyo, 2002. Teori Drama dan Kesusastraan. Institut Kesenian Jakarta (gelesen am . 10 November 2017, um 17.00)

Wahyudi, 2008. Pengantar Teori Sastra. Jakarta: PT. Grasindo

Sugiyono. 2012. MetodeStatistikPenelitian. Jakarta: Rineka Cipta 